\title{
Differential expression of ST6GAL1 in the tumor progression of colorectal cancer
}

\author{
Sen Zhang ${ }^{\text {a, }}{ }^{\text {, Jishun Lu }}{ }^{\text {b, c, } 1}{ }^{\text {, Zhijue Xu }}{ }^{\text {b }}$, Xia Zou ${ }^{\text {a, }}{ }^{b}$, Xue Sun ${ }^{d}$, Yingjiao Xu ${ }^{\text {b }}$, \\ Aidong Shan ${ }^{b}$, Jiaoyang Lu ${ }^{a}$, Xialin Yan ${ }^{a}$, Yalu Cui ${ }^{b}$, Wei Yan ${ }^{b}$, Yuguo Du ${ }^{d}$, Jianguo Gu ${ }^{c,}$, \\ Minhua Zheng a, Bo Feng ${ }^{\text {a, }}{ }^{* *}$, Yan Zhang ${ }^{\text {b, * }}$ \\ a Department of General Surgery, Shanghai Minimally Invasive Surgery Center, Ruijin Hospital, Shanghai Jiao Tong University School of Medicine, 197, Ruijin \\ Er Road, Shanghai 200025, China \\ ${ }^{\mathrm{b}}$ Key Laboratory of Systems Biomedicine (Ministry of Education), and Collaborative Innovation Center of Systems Biomedicine, Shanghai Center for Systems \\ Biomedicine (SCSB), Shanghai Jiao Tong University, 800 Dongchuan Road, Shanghai 200240, China \\ ${ }^{\mathrm{c}}$ Division of Regulatory Glycobiology, Institute of Molecular Biomembrane and Glycobiology, Tohoku Medical and Pharmaceutical University, 4-4-1 \\ Komatsushima, Aobaku, Sendai 981-8558, Miyagi, Japan \\ d State Key Lab of Environmental Chemistry and Ecotoxicology, Research Center for Eco-Environmental Sciences, Chinese Academy of Sciences, Beijing \\ 100085, China \\ e Department of Pharmacology, Pharmacy College, Nantong University, Nantong 226001, Jiangsu, China
}

\section{A R T I C L E I N F O}

\section{Article history:}

Received 16 March 2017

Accepted 31 March 2017

Available online 1 April 2017

\section{Keywords:}

Metabolic labeling

Sialylation

ST6GAL1

Colorectal cancer

\begin{abstract}
A B S T R A C T
Elevated expression of $\beta$-galactoside $\alpha 2,6$-sialyltranferase 1 (ST6GAL1) has been observed in colorectal cancer (CRC) and demonstrated to be important for its tumorigenesis. Here, we found that ST6GAL1 expression was significantly higher in non-metastatic tumors (stage I and II) than that in metastatic tumors (stage III and IV) using 62 pair-matched tumor/normal tissues. To elucidate the molecular mechanisms of how ST6GAL1 affected the CRC progression, we performed a global identification of the substrates of ST6GAL1 in the colon adenocarcinoma cell line SW480. A total of 318 membrane proteins were identified differentially affected by ST6GAL1 overexpression using metabolic labeling and proteomic analysis. Subsequent bioinformatic analysis revealed a list of potential substrates that might mediate the different functions of ST6GAL1 in CRC including cell movement, cell death and survival. Taken together, these results indicate a dynamic change in the expression of ST6GAL1 during the CRC progression and provide a list of sialylated proteins potentially relevant to the different functions of ST6GAL1 in CRC.
\end{abstract}

() 2017 Elsevier Inc. All rights reserved.

\section{Introduction}

Sialic acid is the most abundant terminal monosaccharide of glycoconjugates on the eukaryotic cell surface. It is known to be linked via an $\alpha 2,3$ or $\alpha 2,6$ bond to Gal/GalNAc, or $\alpha 2,8$ bond to sialic acid in proteins. Given the relatively strong electronegative charge

Abbreviations: CRC, colorectal cancer; EGFR, EGF receptor; OE, overexpression; qPCR, quantitative RT-PCR; ST6GAL1, $\beta$-galactoside $\alpha 2,6$-sialyltranferase 1 ; WT, wild-type.

* Corresponding author.

** Corresponding author

E-mail addresses: yanzhang2006@sjtu.edu.cn (Y. Zhang),fengbo2022@163.com (B. Feng).

${ }^{1}$ Both authors contributed equally to this work. of sialic acids and their location at the outmost reaches of the cell surface, sialic acids may regulate numerous cell-cell and cell-matrix interactions. Not surprisingly, altered sialylation has been long associated with tumorigenesis and tumor progression and it is usually driven by the dysregulated expression of certain sialyltransferases and sialidases responsible for the addition and removal of sugars [1-3].

Among these, $\beta$-galactoside $\alpha 2,6$-sialyltranferase 1 (ST6GAL1), which primarily produces $\alpha 2,6$ linked sialic acids on $N$-glycans, has received much attention in recent years as elevated expression of this sialyltransferase was observed in multiple types of carcinomas including colorectal, ovarian, breast, prostate, ovarian, and pancreatic carcinomas [4]. The selective enrichment of ST6GAL1mediated $\alpha 2,6$ sialic acids on tumor cells is significant in that it may elicit various biologic outcomes. It has been also reportedly 
involved in tumorigenesis, epithelial mesenchymal transition, cell adhesion, migration and invasion, apoptosis, drug resistance, immune evasion and maintenance of cancer stem cell property [4-10]. In contrast to these important roles, there is limited information regarding the molecular details of how ST6GAL1-catalyzed $\alpha 2,6$ sialylated proteins mediate these functions [11]. ST6GAL1 could regulate the cell migration and invasion partly by increasing the $\alpha 2,6$ sialylation of $\beta 1$ integrin [12-14]. Sialylation of the Fas death receptor by ST6GAL1 provides protection against Fasmediated apoptosis in colon carcinoma cells [15]. In addition, there are also several other reported proteins whose activities are regulated by sialylation which includes CD45, EGFR, and PECAM [12-19]. Clearly, these limited proteins could not provide the comprehensive explanation for its diverse roles in tumors. To obtain a better understanding of the mechanistic roles of this sialyltransferase, identification of its additional function-related sialylated proteins is of critical importance.

The up-regulated expression of ST6GAL1 was first described in colorectal cancer (CRC) [20]. Increasing evidence has indicated that ST6GAL1 may serve as a major inhibitor of several cell death pathways and contribute to the colorectal tumorigenesis [6,15,21]. However, the mechanistic roles of ST6GAL1 in the tumor progression are still obscure. In the current study, interestingly, we found that ST6GAL1 was significantly induced in tumors at stage I and II but not in those at stage III and IV when compared with their paired normal tissues. Subsequent metabolic labeling and proteomics analysis in the colon cancer cell line SW480 revealed a list of proteins that may mediate the effects of ST6GAL1 on tumor development and progression. These results indicate a dynamic change in ST6GAL1 expression in human CRC progression and suggest the distinct roles of ST6GAL1 at different stages.

\section{Materials and methods}

Tissue Specimens - From May 2009 to Jun 2012, a total of 62 CRC cases with pathological stage I $(\mathrm{n}=19)$, II $(\mathrm{n}=20)$, III $(\mathrm{n}=17)$ and IV $(n=6)$ were recruited from Ruijin Hospital (Shanghai, China) in accordance with the guidelines set by the Ethical Committee of Ruijin Hospital. All participants gave the written, informed consent and no participants had received any medication prior to sample collection. Staging of CRC was performed according to the Union Internationale Contre Le Cancer (UICC). Tumor tissues and paired normal colonic tissues, located approximately $10 \mathrm{~cm}$ from the distal edge of the tumor were snap frozen in liquid nitrogen 30 min after vessel ligation and stored in the liquid nitrogen prior to analysis. The inclusion and exclusion criteria of patients, and protein extraction were performed as described in Ref. [22].

Cell Lines and Cell Culture - The HEK 293T and SW480 cell lines were purchased from ATCC (Manassas, VA, USA). The HEK 293T cells were cultured as described previously [10]. SW480 and ST6GAL1-overexpressing cells were maintained in Leibovitz's L15 Medium (HyClone, Logan, Utah, USA) with 10\% FBS under a humidified atmosphere at $37{ }^{\circ} \mathrm{C}$ without $\mathrm{CO}_{2}$ addition. The ST6GAL1overexpressing cells were established by virus transfection and cell-sorting using FACS Aria II (BD Bioscience, Bedford, Massachusetts, USA).

Western Blot Analysis and Immunoprecipitation - Western blot analysis and immunoprecipitation were performed as described previously [10]. For details, please refer to our supplementary materials.

Flow Cytometry Analysis of Cells - Cell cytometry was performed as reported in Ref. [23]. Cells were grown to about 90\% confluency and detached using trypsin containing $1 \mathrm{mM}$ EDTA at $37{ }^{\circ} \mathrm{C}$, and washed three times with cold PBS. Then, cells were stained with $1 \mu \mathrm{g} / \mathrm{ml}$ Alkyne 555 (Invitrogen, Eugene, Oregon, USA) for $30 \mathrm{~min}$ at RT temperature or with $10 \mu \mathrm{g} / \mathrm{ml}$ SNA-FITC (Vector, Burlingame, California, USA) or $10 \mu \mathrm{g} / \mathrm{ml}$ MAA-FITC (EY Laboratories, San Mateo, California, USA) for 60 min on ice. Finally, cells were washed three times with PBS and analyzed by flow cytometry (BD Biosciences, San Jose, California, USA).

qPCR for mRNA Expression Analysis - The qPCR analysis was performed as described previously [24]. The sequences of primers used for quantitative RT-PCR (qPCR) analysis are as follows: GAPDH (5'-TTCAACAGCAACTCCCACTCTT-3' and 5'-TGGTCCAGGGTTTCTTACTCC- $\left.3^{\prime}\right)$, EGFR (5'-ACCTGCGTGAAGAAGTGTCC- $3^{\prime}$ and $5^{\prime}-$ CGTCTTCCTCCATCTCATAGC- $\left.3^{\prime}\right), \quad$ ABCB1(5'-GCCAAAGCCAAAATATCAGC- $3^{\prime}$ and $5^{\prime}$-TTCCAATGTGTTCGGCAT- $3^{\prime}$ ).

Cell Migration Assay - Cell migratory ability was examined with Transwell (BD BioCoat ${ }^{\mathrm{TM}}$ control inserts, 8.0-mm inserts; BD Biosciences, Bedford, Massachusetts, USA) as described previously [23]. Transwells were coated only on the bottom side with $10 \mu \mathrm{g} / \mathrm{ml}$ fibronectin or collagen I at $4{ }^{\circ} \mathrm{C}$ overnight.

Metabolite Labeling and Fluorescence Detection - cells $\left(4.0 \times 10^{6}\right)$ were cultured with $100 \mu \mathrm{M}$ of the $\mathrm{Ac}_{4} \mathrm{ManNAz}$ or ManNAc for $60 \mathrm{~h}$. After metabolic labeling, the cells were incubated with Alkyne 555, catalyst (CuSO4, NaVc, TBTA) for click chemistry [25]. The obtained cells were then detected with a laser-scanning confocal microscope (Nikon, Tokyo, Japan) or flow cytometry (BD Biosciences, San Jose, California, USA) for the check of the labeling efficiency. For details, please refer to [26].

Protein Enrichment and Mass Spectrometry Analysis - After washing with PBS, $1 \times 10^{7}$ metabolic labeled cells were used to perform the subsequent click reaction with biotinylated alkyne and glycoprotein enrichment by streptavidin-agarose resin as described previously [25]. The beads were pelleted by centrifugation and washed, reduced and alkylated followed by on bead trypsin digestion as shown in Ref. [27]. The released peptides were analyzed on a Q Exactive Plus mass spectrometer equipped with an Easy-nLC 1000 (Thermo Fisher Scientific, Odense, Denmark). Spectra was searched against a human proteins database (Uniprot Complete Proteome - taxonomy Homo sapiens, release 2016.08) using the Andromeda module of MaxQuant software v. 1.5.5.1. For details, please refer to our supplementary materials.

Bioinformatic Annotation and Analysis - $\mathrm{N}$ - and $\mathrm{O}$-glycoprotein prediction analysis using the NetNGlyc 1.0 and the NetOGlyc 4.0 servers was performed. Gene ontology (GO) enrichment analysis was carried out using DAVID Bioinformatics Resources 6.8. Functional analysis was generated using the Ingenuity Pathways Analysis (IPA) software v7.1 (Ingenuity Systems).

\section{Results}

To explore the roles of ST6GAL1 in the CRC progression, we first evaluated its expression in the tissue specimens at different stages by western blot analysis (Fig. $1 \mathrm{~A}$ and S1). 62 patients were randomly selected based only on the criteria of matched age and gender (Table 1). Interestingly, the expression of ST6GAL1 was significantly increased in tumors at the stage I and II as compared with their pair-matched normal tissues, while no statistical difference was observed when the tumors were developed to the stage III and IV (Fig. 1B). Furthermore, the expression of ST6GAL1 in tumors at stage I and II was significantly higher than that at stage III and IV (Fig. 1C). These results clearly indicate a dynamic change in ST6GAL1 expression in the colorectal tumors at different stages, suggesting important roles of ST6GAL1 in CRC progression.

Next, to explore molecular mechanisms of how ST6GAL1 affected the CRC progression, we first overexpressed this sialyltransferase in SW480, a human colon adenocarcinoma cell line with the low expression of ST6GAL1. Western blot, lectin blot, cell cytometry and qPCR results demonstrated the high expression of 
A

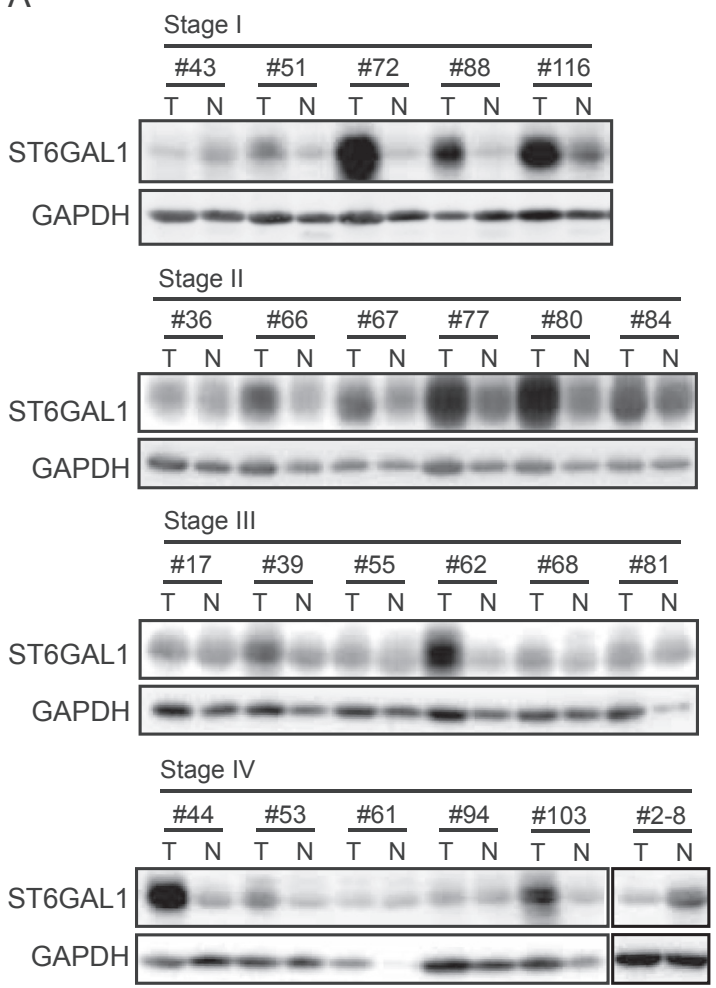

B

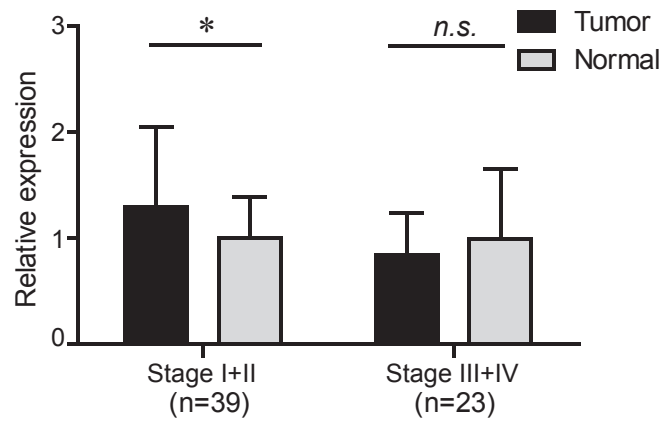

C

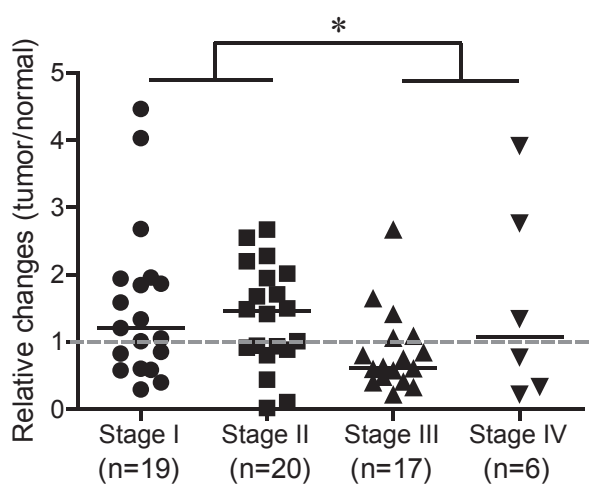

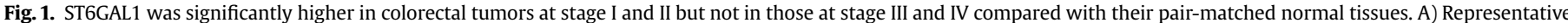

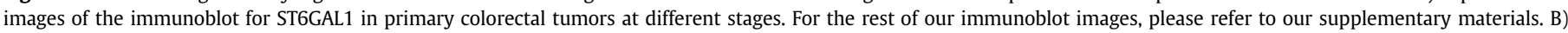

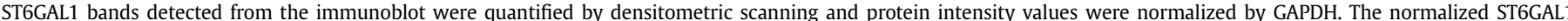

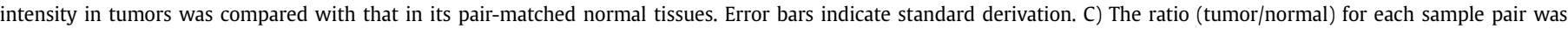
calculated. Middle lines indicate Median values (n.s.: no significance; ${ }^{*}, p<0.05$ by paired $t$-test or Mann-Whitney $U$ test).

Table 1

Clinical features of samples used in this study.

\begin{tabular}{|c|c|c|c|c|}
\hline UICC Stage & Stage I & Stage II & Stage III & Stage IV \\
\hline Number & 19 & 20 & 17 & 6 \\
\hline \multicolumn{5}{|l|}{ Age (year) } \\
\hline median/range & $59 / 47-76$ & $60 / 50-78$ & $59 / 40-77$ & $61 / 35-74$ \\
\hline \multicolumn{5}{|l|}{ Gender } \\
\hline Male & 11 & 13 & 10 & 3 \\
\hline Female & 8 & 7 & 7 & 3 \\
\hline \multicolumn{5}{|l|}{ Location ${ }^{a}$} \\
\hline $\mathrm{AC}$ & 4 & 4 & 3 & - \\
\hline TC & 1 & - & - & - \\
\hline DC & 2 & 4 & - & 2 \\
\hline SC & 4 & 2 & 1 & 1 \\
\hline $\mathrm{RC}$ & 8 & 10 & 13 & 3 \\
\hline \multicolumn{5}{|l|}{ CEA (ng/ml) } \\
\hline median/range & $2.62 / 1.36-8.90$ & $4.19 / 0.94-376.43$ & $2.86 / 0.7-891.16$ & $10.43 / 4.43-303.24$ \\
\hline \multicolumn{5}{|l|}{ CA19-9 (ng/ml) } \\
\hline median/range & $6.53 / 2.00-42.70$ & $11.42 / 0.80-91.70$ & $18.9 / 2.00-1554.6$ & $185.35 / 51.60-3770.46$ \\
\hline \multicolumn{5}{|l|}{ CA125 (ng/ml) } \\
\hline median/range & $10.00 / 3.9-32.6$ & $14.80 / 7.40-34.90$ & $13.60 / 7.20-62.30$ & $84.55 / 14.80-238.80$ \\
\hline \multicolumn{5}{|l|}{$\mathrm{AFP}(\mathrm{ng} / \mathrm{ml})$} \\
\hline median/range & $2.96 / 1.48-4.39$ & $3.15 / 1.57-6.14$ & $3.19 / 1.89-8.40$ & $5.42 / 4.88-5.88$ \\
\hline
\end{tabular}

a AC, ascending colon; TC, transverse colon; DC, descending colon; SC, sigmoid colon; RC, rectum.

ST6GAL1 in the ST6GAL1-overexpressing SW480 cells (Fig. 2A-D). Then, to identify the sialylated proteins differentially affected by ST6GAL1, SW480 and ST6GAL1-overexpressing cells were separately labeled with $\mathrm{Ac}_{4} \mathrm{ManNAz}$ and ManNAc followed by the enrichment and identification of the sialylated membrane proteins. Fig. 3A showed the schematic representation of our strategy.
$\mathrm{Ac}_{4} \mathrm{ManNAz}$ is intracellularly deacetylated and used to incorporate azidos to tag the sialic acid linked glycans and the sialylated proteins [26]. The azido-tagged proteins on the cell surface were then reacted with biotin-alkyne and enriched with streptavidin-beads. The sialylated proteins were efficiently and specifically labeled by azido sugars, as shown by the strong signals in the glycoproteins 
A
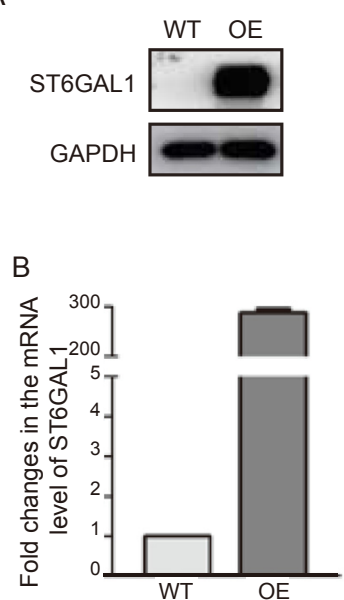

C

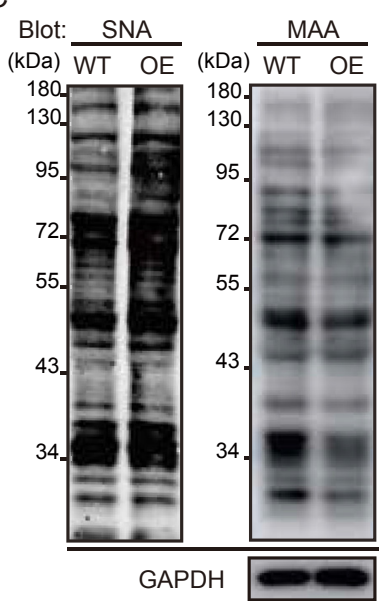

D

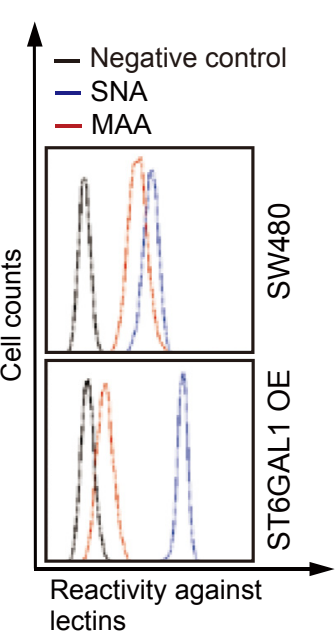

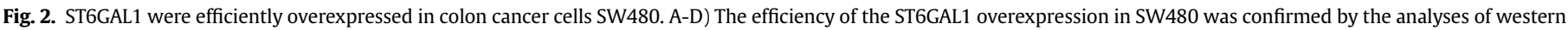
blot, qPCR, lectin blot and cell cytometry. GAPDH was used as an internal control.

A

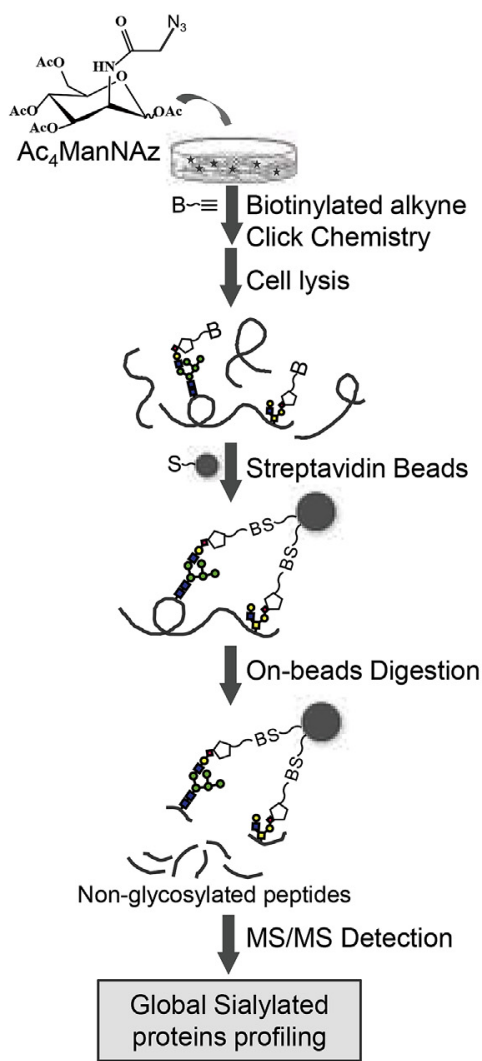

B
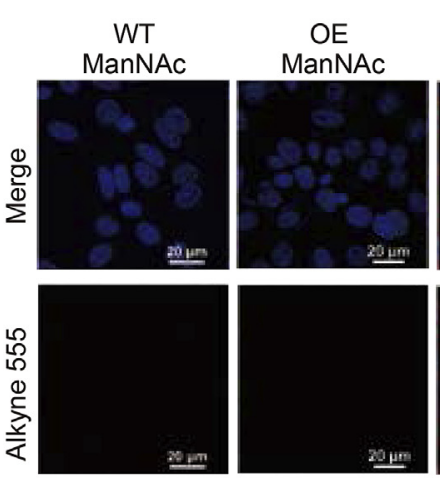

$\mathrm{D}$

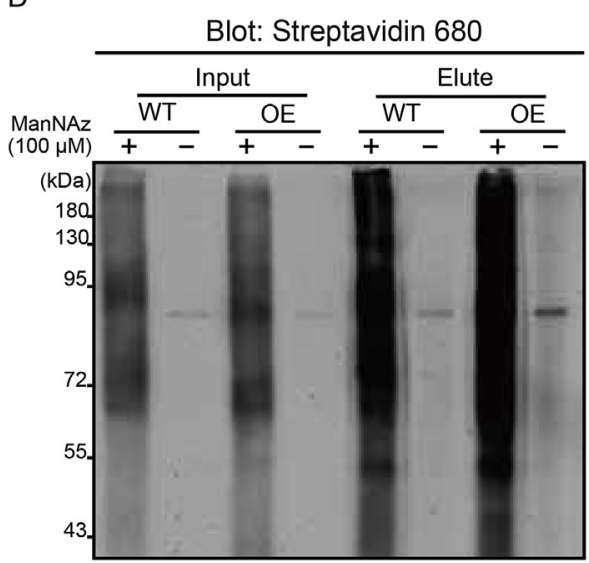

$\mathrm{C}$

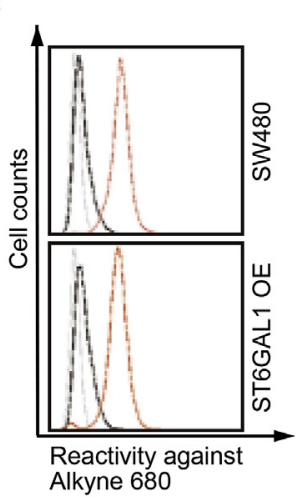

$\mathrm{E}$

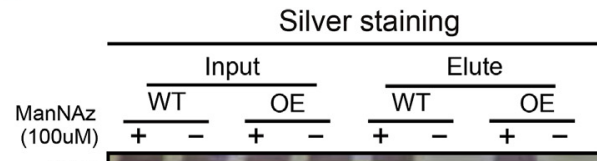

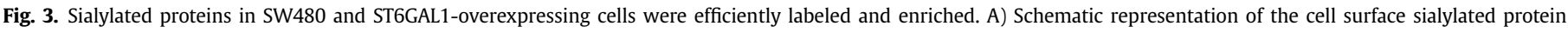

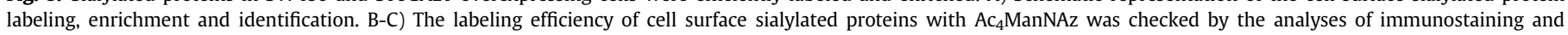
cytometry. D-E) The selectivity and efficiency of the enrichment of these proteins were examined by the analyses of silver staining and western blot with streptavidin 680 .

from the cells incubated with $\mathrm{Ac}_{4} \mathrm{ManNAz}$ but not from those pretreated with ManNAc according to the fluorescence-staining and FACS analyses (Fig. 3B and C). We also checked the efficiency of sialylated protein enrichment by silver staining and western blot analyses. The amount of proteins as well as the signals against streptavidin 680 in the elute fraction of $\mathrm{Ac}_{4} \mathrm{ManNAz}$ treated cells were markedly higher as compared with those incubated with ManNAc (Fig. 3D and E), indicating the efficient and selective enrichment of the azido sugar modified proteins.

The MS analysis of the elute fractions collected from the cells 
cultured with Ac 4 ManNAz led to the identification of a total of 709 membrane proteins by searching the data against the Uniprot Complete Proteome Human Database and GO analysis (Cellular Component). Prediction analysis of these proteins indicated that 567 of them could be $N$-glycosylated and 625 of them could be $O$ glycosylated (Fig. 4A). Considering sialic acids mainly exist as the terminal sugars on the $\mathrm{N}$-glycans, $\mathrm{O}$-glycans and glycolipids, these results suggest that most of the labeled glycoproteins arising from the $\mathrm{Ac}_{4} \mathrm{ManNAz}$ treatment might be sialylated.

Next, we examined the differential regulation of glycoproteins upon ST6GAL1 overexpression in SW480 cells based on our identified set of 709 membrane proteins. Proteins were considered as differentially expressed between the wild-type SW480 and ST6GAL1-overexpressing cells if their fold change in relative expression $\geq 2$ (47-49). Based on these selection criteria, we found a total of 318 glycoproteins were differentially affected (up-regulated or down-regulated or specifically expressed in either cells) after
ST6GAL1 overexpression in SW480. Volcano plot-log10 (intensity of the protein in ST6GAL1 overexpression) vs. $\log 2$ (fold change of ST6GAL1 overexpression/wild-type) was constructed to graphically display the quantitative data using the overlapped proteins between control and ST6GAL1-overexpressing cells (Fig. 4B). To evaluate the potential roles of ST6GAL1 in CRC, these proteins were used for the functional analysis by the IPA software. Fig. 4C showed the top 15 most significantly perturbed functions upon the overexpression of ST6GAL1 in SW480. The first three were cell movement, cell death and survival, and cell growth and proliferation, which are in good agreement with numerous reports on the functions of ST6GAL1 under pathological conditions [4]. Table S1 listed the proteins that are related with these functions.

To experimentally confirm these changes, two proteins $A B C B 1$ ( $\sim 10$ folds up) and EGFR ( 2 folds up), which showed big difference in the extent of their changes, were selected to perform the qPCR, immunoprecipitation and western blot analyses (Fig. 4D-F). ABCB1
A

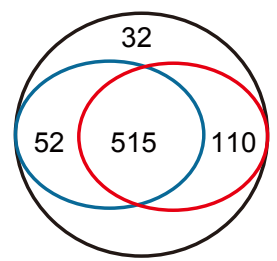

Total proteins

Predicted $\mathrm{N}$-glycosylated proteins

Predicted O-glycosylated proteins

B

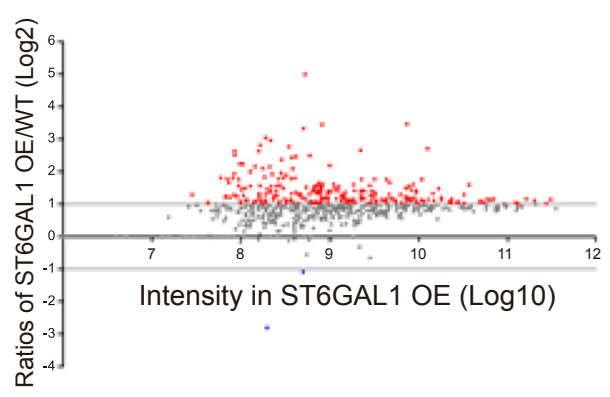

D

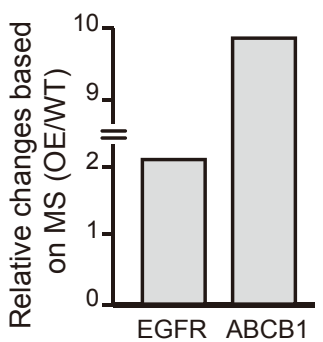

C - Log ( $p$-value)

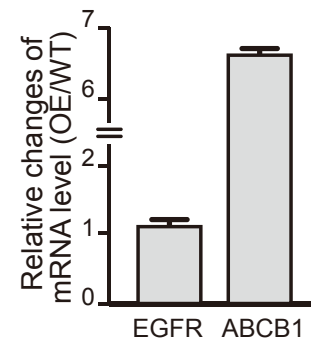

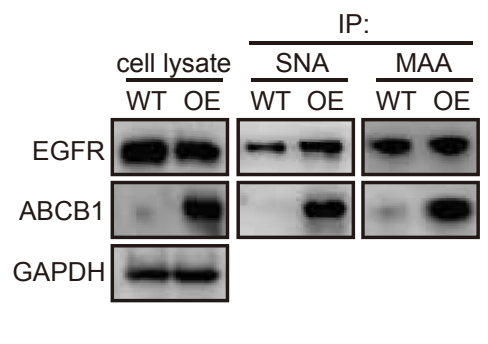

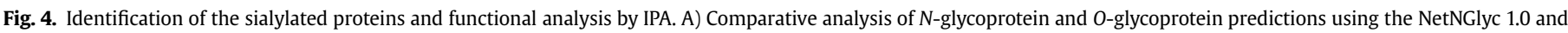

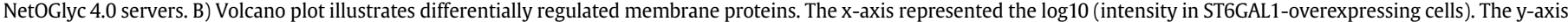

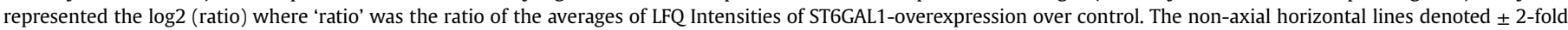

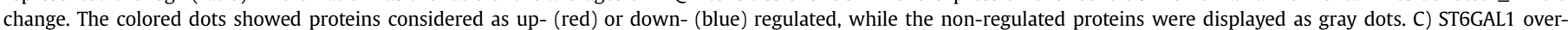

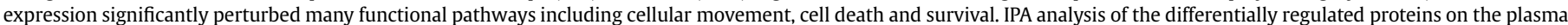

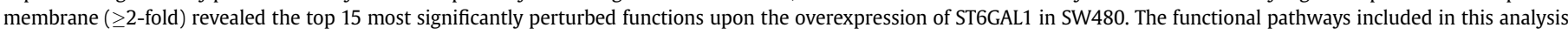

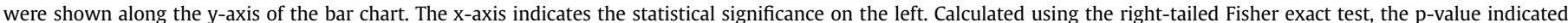

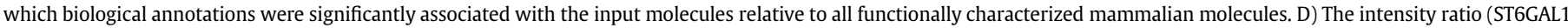

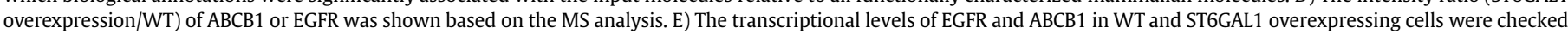

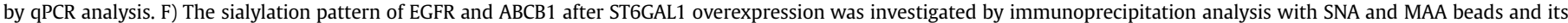

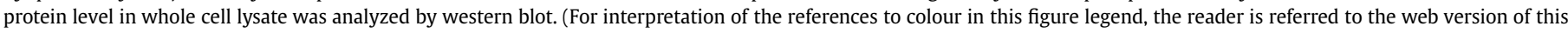
article.) 
were significantly upregulated at both mRNA and protein levels, indicating the different expression of this protein observed in our MS data is mainly due to its increased mRNA level. In contrast, there was little change in either mRNA or protein level of EGFR after ST6GAL1 overexpression. Since immunoprecipitation analysis showed a significant increase in the $\alpha 2,6$ sialylation level, it is highly likely that $\sim 2$ folds upregulation of EGFR observed in our MS data is attributed to its enhanced sialylation.

\section{Discussion}

Elevated expression of ST6GAL1 has been observed in multiple types of carcinomas, among which colorectal cancer was the first reported [20]. Although it has been well documented that ST6GAL1 expression contributes to the colorectal tumorigenesis [6], the mechanistic roles of this protein in tumor progression are still obscure. Interestingly, we found that as compared with their pairmatched normal tissues, the expression of ST6GAL1 was significantly increased in tumors at stage I and II (non-metastatic), but not at stage III and IV (metastatic). Meanwhile, ST6GAL1 expression in the tumors at stage I and II was statistically higher than that at stage III and IV, suggesting a dynamic change of ST6GAL1 expression in CRC progression. Metabolic labeling revealed 318 sialylated membrane proteins differentially affected by ST6GAL1 overexpression. Considering the distinct roles of different linkages of sialylation in cell biology, these results suggest important roles of the dynamic change in ST6GAL1 expression during the CRC progression.

We showed that high expression of ST6GAL1 was decreased at the advanced stage of CRC. Consistent with our observation, there is evidence that the mRNA level of ST6GAL1 is significantly increased in colorectal carcinoma specimens, but not in highly invasive tumors and tumors exhibiting distant metastases when compared with their pair-matched normal tissues [28]. These results suggest that high expression of this enzyme may not benefit the CRC progression to the advanced/metastatic stage. Supporting this hypothesis, a very recent work showed that knockdown of ST6GAL1 dramatically enhances the migration and invasion of the colon cancer cell line SW620, and also promotes its liver metastasis in vivo [29]. Furthermore, our MS data showed that ST6GAL1 overexpression increased the expression of ICAM-1, a liver metastasis suppressor of colon cancer (data not shown). It is well-known that sialyl Lewis structure serves as a key mechanism for directing tumor cell adhesion to the endothelium and the $\alpha 2,3$ linked sialic acid on sialyl Lewis structure is critical for this function. A previous report showed that $\alpha 2,3$ sialylated type 2 chain structures is predominantly expressed in colorectal tissues associated with the malignant transformation, in particular, with the lymphatic spread of distal colorectal adenocarcinoma [30]. Therefore, it is possible that decreased expression of ST6GAL1 and $\alpha 2,6$ sialylation may benefit the tumor metastasis by increasing the $\alpha 2,3$ sialylated structures on cell surface because $\alpha 2,3$ and $\alpha 2,6$ sialyltranferases share the common glycan substrates.

Our IPA analysis revealed a list of novel glycoproteins that could mediate these functions above. Among the list, we found that overexpression of ST6GAL1 resulted in an increased transcription of ABCB1 by more than $\sim 6$ folds. Consistent with this, it has been previously reported that ST6GAL1 expression could induce the expression of $A B C B 1$ in human leukemia cells [31]. In addition, a recent study showed that KAI1 is significantly decreased after ST6GAL1 knockdown in SW620 cells. This protein could also be found in our list, which was increased by $\sim 2$-fold after ST6GAL1 overexpression. These results partially suggest the reliability of the list we obtained, which may contribute to the identification of additional molecules linking the functions of ST6GAL1 in tumor.

\section{Declaration}

We declare that this manuscript is original, has not been published before and is not currently being considered for publication elsewhere. There is no conflict of interest concerning this manuscript.

\section{Acknowledgement}

This work was supported by The National Natural Science Foundation of China (31370806, 31170771, 31600643, 31670807), The National Basic Research Program of China (2012CB822103, 2011CB910603), Shanghai Municipal Commission of Health and Family Planning Program (201640030), Shanghai Jiao Tong University Interdiscipline with Medicine Program (YG2013MS26), China Postdoctoral Science Foundation (2016M600310).

\section{Appendix A. Supplementary data}

Supplementary data related to this article can be found at http:// dx.doi.org/10.1016/j.bbrc.2017.03.167.

\section{References}

[1] A. Harduin-Lepers, M.-A. Krzewinski-Recchi, F. Colomb, F. Foulquier, S. GrouxDegroote, P. Delannoy, Sialyltransferases functions in cancers (Elite edition), Front. Biosci. 4 (2011) 499-515.

[2] F. Dall'Olio, N. Malagolini, M. Trinchera, M. Chiricolo, Sialosignaling: sialyltransferases as engines of self-fueling loops in cancer progression, Biochimica Biophysica Acta (BBA) - General Subj. 1840 (2014) 2752-2764.

[3] S.S. Pinho, C.A. Reis, Glycosylation in cancer: mechanisms and clinical implications, Nat. Rev. Cancer 15 (2015) 540-555.

[4] J. Lu, J. Gu, Significance of beta-galactoside alpha2,6 sialyltranferase 1 in cancers, Molecules 20 (2015) 7509-7527.

[5] X. Chen, L. Wang, Y. Zhao, S. Yuan, Q. Wu, X. Zhu, B. Niang, S. Wang, J. Zhang, ST6Gal-I modulates docetaxel sensitivity in human hepatocarcinoma cells via the p38 MAPK/caspase pathway, Oncotarget 7 (2016) 51955-51964.

[6] M.J. Schultz, A.T. Holdbrooks, A. Chakraborty, W.E. Grizzle, C.N. Landen, D.J. Buchsbaum, M.G. Conner, R.C. Arend, K.J. Yoon, C.A. Klug. D.C. Bullard, R.A. Kesterson, P.G. Oliver, A.K. O'Connor, B.K. Yoder, S.L. Bellis, The tumorassociated glycosyltransferase ST6Gal-I regulates stem cell transcription factors and confers a cancer stem cell phenotype, Cancer Res. 76 (2016) 3978-3988.

[7] Z. Liu, A.F. Swindall, R.A. Kesterson, T.R. Schoeb, D.C. Bullard, S.L. Bellis, ST6Gal-I regulates macrophage apoptosis via alpha2-6 sialylation of the TNFR1 death receptor, J. Biol. Chem. 286 (2011) 39654-39662.

[8] M. Perdicchio, J.M. Ilarregui, M.I. Verstege, L.A. Cornelissen, S.T. Schetters, S. Engels, M. Ambrosini, H. Kalay, H. Veninga, J.M. den Haan, L.A. van Berkel, J.N. Samsom, P.R. Crocker, T. Sparwasser, L. Berod, J.J. Garcia-Vallejo, Y. van Kooyk, W.W. Unger, Sialic acid-modified antigens impose tolerance via inhibition of T-cell proliferation and de novo induction of regulatory T cells, Proc. Natl. Acad. Sci. U. S. A. 113 (2016) 3329-3334.

[9] C.M. Britain, K.A. Dorsett, S.L. Bellis, The glycosyltransferase ST6Gal-I protects tumor cells against serum growth factor withdrawal by enhancing survival signaling and proliferative potential, J. Biol. Chem. 292 (2017) 4663-4673.

[10] J. Lu, T. Isaji, S. Im, T. Fukuda, N. Hashii, D. Takakura, N. Kawasaki, J. Gu, betaGalactoside alpha2,6-sialyltranferase 1 promotes transforming growth factorbeta-mediated epithelial-mesenchymal transition, J. Biol. Chem. 289 (2014) 34627-34641.

[11] M.J. Schultz, A.F. Swindall, S.L. Bellis, Regulation of the metastatic cell phenotype by sialylated glycans, Cancer Metastasis Rev. 31 (2012) 501-518.

[12] E.C. Seales, G.A. Jurado, B.A. Brunson, J.K. Wakefield, A.R. Frost, S.L. Bellis, Hypersialylation of beta1 integrins, observed in colon adenocarcinoma, may contribute to cancer progression by up-regulating cell motility, Cancer Res. 65 (2005) 4645-4652.

[13] F.M. Shaikh, E.C. Seales, W.C. Clem, K.M. Hennessy, Y. Zhuo, S.L. Bellis, Tumor cell migration and invasion are regulated by expression of variant integrin glycoforms, Exp. Cell Res. 314 (2008) 2941-2950.

[14] D.R. Christie, F.M. Shaikh, J.A.t. Lucas, J.A. Lucas 3rd, S.L. Bellis, ST6Gal-I expression in ovarian cancer cells promotes an invasive phenotype by altering integrin glycosylation and function, J. Ovarian Res. 1 (2008) 3.

[15] A.F. Swindall, S.L. Bellis, Sialylation of the Fas death receptor by ST6Gal-I provides protection against Fas-mediated apoptosis in colon carcinoma cells, J. Biol. Chem. 286 (2011) 22982-22990.

[16] M. Amano, M. Galvan, J. He, L.G. Baum, The ST6Gal I sialyltransferase selectively modifies $\mathrm{N}$-glycans on CD45 to negatively regulate galectin-1-induced CD45 clustering, phosphatase modulation, and T cell death, J. Biol. Chem. 
278 (2003) 7469-7475.

[17] H.Y. Yen, Y.C. Liu, N.Y. Chen, C.F. Tsai, Y.T. Wang Y.J Chen, T.L. Hsu, P.C. Yang C.H. Wong, Effect of sialylation on EGFR phosphorylation and resistance to tyrosine kinase inhibition, Proc. Natl. Acad. Sci. U. S. A. 112 (2015) 6955-6960.

[18] J.J. Park, J.Y. Yi, Y.B. Jin, Y.J. Lee, J.S. Lee, Y.S. Lee, Y.G. Ko, M. Lee, Sialylation of epidermal growth factor receptor regulates receptor activity and chemosensitivity to gefitinib in colon cancer cells, Biochem. Pharmacol. 83 (2012) 849-857.

[19] S. Kitazume, R. Imamaki, K. Ogawa, Y. Komi, S. Futakawa, S. Kojima, Y. Hashimoto, J.D. Marth, J.C. Paulson, N. Taniguchi, Alpha2,6-sialic acid on platelet endothelial cell adhesion molecule (PECAM) regulates its homophilic interactions and downstream antiapoptotic signaling, J. Biol. Chem. 285 (2010) 6515-6521.

[20] F. Dall'Olio, N. Malagolini, G. di Stefano, F. Minni, D. Marrano, F. Serafini-Cessi, Increased CMP-NeuAc:Gal beta 1,4GlcNAc-R alpha 2,6 sialyltransferase activity in human colorectal cancer tissues, Int. J. Cancer 44 (1989) 434-439.

[21] Y. Zhuo, S.L. Bellis, Emerging role of $\alpha 2,6$-sialic acid as a negative regulator of galectin binding and function, J. Biol. Chem. 286 (2011) 5935-5941.

[22] X. Zou, B. Feng, T. Dong, G. Yan, B. Tan, H. Shen, A. Huang, X. Zhang, M. Zhang, P. Yang, M. Zheng, Y. Zhang, Up-regulation of type I collagen during tumorigenesis of colorectal cancer revealed by quantitative proteomic analysis, J. Proteomics 94 (2013) 473-485.

[23] J. Lu, T. Isaji, S. Im, T. Fukuda, A. Kameyama, J. Gu, Expression of N-Acetylglucosaminyltransferase III suppresses alpha2,3-sialylation, and its distinctive functions in cell migration are attributed to alpha2,6-sialylation levels, J. Biol. Chem. 291 (2016) 5708-5720.

[24] Y. Xu, W. Pang, J. Lu, A. Shan, Y. Zhang, Polypeptide N-
Acetylgalactosaminyltransferase 13 contributes to neurogenesis via stabilizing the mucin-type O-Glycoprotein podoplanin, J. Biol. Chem. 291 (2016) 23477-23488.

[25] S. Wang, W. Xie, X. Zhang, X. Zou, Y. Zhang, Disulfide- and terminal alkynefunctionalized magnetic silica particles for enrichment of azido glycopeptides, Chem. Commun. (Camb) 48 (2012) 5907-5909.

[26] J. Du, S. Hong, L. Dong, B. Cheng, L. Lin, B. Zhao, Y.G. Chen, X. Chen, Dynamic sialylation in transforming growth factor-beta (TGF-beta)-induced epithelial to mesenchymal transition, J. Biol. Chem. 290 (2015) 12000-12013.

[27] R. Sheta, F. Roux-Dalvai, C.M. Woo, F. Fournier, S. Bourassa, C.R. Bertozzi, A. Droit, D. Bachvarov, Proteomic dataset for altered glycoprotein expression upon GALNT3 knockdown in ovarian cancer cells, Data Brief. 8 (2016) 342-349.

[28] T. Petretti, W. Kemmner, B. Schulze, P.M. Schlag, Altered mRNA expression of glycosyltransferases in human colorectal carcinomas and liver metastases, Gut 46 (2000) 359-366.

[29] Y.R. Jung, J.J. Park, Y.B. Jin, Y.J. Cao, M.J. Park, E.J. Kim, M. Lee, Silencing of ST6Gal I enhances colorectal cancer metastasis by down-regulating KAI1 via exosome-mediated exportation and thereby rescues integrin signaling, Carcinogenesis 37 (2016) 1089-1097.

[30] T. Fukasawa, T. Asao, H. Yamauchi, M. Ide, Y. Tabe, T. Fujii, S. Yamaguchi, S. Tsutsumi, S. Yazawa, H. Kuwano, Associated expression of $\alpha 2,3$ sialylated type 2 chain structures with lymph node metastasis in distal colorectal cancer, Surg. Today 43 (2013) 155-162.

[31] H. Ma, L. Cheng, K. Hao, Y. Li, X. Song, H. Zhou, L. Jia, Reversal effect of ST6GAL 1 on multidrug resistance in human leukemia by regulating the PI3K/Akt pathway and the expression of P-gp and MRP1, PLoS One 9 (2014) e85113. 